\title{
Large Grain Niobium Cavity R\&D In Asia and the Future
}

\author{
K. Saito, F.Furuta, T.Saeki, H.Inoue, J.Shim ${ }^{1}$, J.Ahn ${ }^{1}$. E.S.Kim ${ }^{2}$, Q.Xu ${ }^{3}$, \\ Z.Zong ${ }^{3}$, J.Gao ${ }^{3}$, P.Kneisel ${ }^{4}$ and G. R. Myneni ${ }^{4}$ \\ KEK, Tsukuba Ibaraki-ken, Japan \\ ${ }^{\prime}$ Pusan University, Pusan, South Korea \\ ${ }^{2}$ Kynongpook University, Daegu,South Korea \\ ${ }^{3}$ IHEP, Beijing, China \\ ${ }^{4} J e f f e r s o n$ Lab, 12000 Jefferson Ave, Newport News, VA 23606
}

\begin{abstract}
The status of the large grain niobium cavity R\&D in Asia and the future scope are presented. Recently KEK has received CBMM and NingXia large grain niobium sheets through collaborations. KEK has fabricated $1.3 \mathrm{GHz}$ single cell cavities using these materials and measured the cavity performance. Those results are presented in this paper.
\end{abstract}

Keywords: Superconducting cavities, niobium.

PACS: 85.25-j, 74-70.-b

\section{INTRODUCTION}

Application of large grain niobium to SRF cavity fabrication was started for two reasons two years ago: to push the current gradient limitation in particular on buffered chemical polished (BCP) polycrystalline niobium cavities, and a programmatic reason of cost effective niobium production for a large scale SRF application like ILC or EURO-XFEL. Currently it is a firm fact that BCP and Electropolishing (EP) both bring a Q-slope around $\mathrm{E}_{\mathrm{acc}} \sim 25 \mathrm{MV} / \mathrm{m}$ if no baking is carried out during vacuum evacuation. Effect of the baking looks different between BCP and EP. In case of EP, $\mathrm{Q}$-slope is perfectly eliminated and the gradient often reaches the critical RF limitation, typically $40 \mathrm{MV} / \mathrm{m}$ on TESLA shape or $50 \mathrm{MV} / \mathrm{m}$ on LL shape. In case of $\mathrm{BCP}$, the Q-slope is recovered rather but not perfectly and the gradient is usually limited to a lower value than the critical field, typically $30 \mathrm{MV} / \mathrm{m}$. BCP has a feature to produce sharp steps at grain boundaries due to chemical etching process. On the other hand, EP makes smooth surface even at the grain boundaries. The different baking effect suggests that grain boundary makes a harmful influence, such as magnetic field enhancement on the high gradient performance. Investigation of large grain/single crystal niobium could bring additional information on this subject.

The production process is rather simplified in large grain niobium sheets with a diameter $<300 \mathrm{~mm}$. Niobium sheet $(270-260 \mathrm{~mm}$ diameter) for $1.3 \mathrm{GHz}$ cavity can be produced by directly slicing a large grain ingot. Forging and rolling processes in current production are eliminated. So far, several methods have been used for the ingot

\section{CP927, Single Crystal Large Grain Niobium Technology, International Niobium Workshop edited by G. R. Myneni, T. Carneiro, and A. Hutton \\ 2007 American Institute of Physics 978-0-7354-0437-3/07/\$23.00}


slicing: electron discharge machining (EDM), mechanical sawing (MS), and wire sawing like silicon wafer production. However, EDM is time consuming and MS has lots of waste material. In the ILC project, we need $\sim 500$ sheets per day for three years cavity production If a fast slicing machine is developed, a large cost reduction can be expected.

So far, JLAB and DESY are going ahead on large grain/single crystal cavity R\&D. P.Kneisel and G.Rao have introduced this method and demonstrated very promising results on BCP cavities. KEK has used EP routinely for polycrystalline cavities and reached high gradient. Large grain cavity was not a critical issue to KEK except for lowering the cost. However, we have recently started an R\&D program with the help of P.Kneisel at JLAB, USA and J.Gao at IHEP, China, to evaluate the advantages of EP on large grain cavity. We have fabricated and tested four $1.3 \mathrm{GHz}$ single cell cavities. We present these results in this paper. It should be emphasized that KEK is still at very early stage on this $R \& D$.

\section{CURRENT R\&D SCHEME OF THE LARGE GRAIN NIOBIUM CAVTTY}

At first, we make a brief review on large/single crystalline niobium cavity R\&D over the world.

\section{USA}

As seen in Fig.1, which shows the R\&D scheme on large grain/single crystal niobium cavity development, four niobium production firms can produce large grain ingot: CBMM in Brazil, Wah Chang in USA, WC Heraeus in Germany, and NingXia in China. P. Kneisel et al. have investigated CBMM, Wah Chang, and NingXia large grain materials on $\mathrm{BCP}$ and showed very promising results [1]. They have demonstrated a very narrow performance scatter between those three different materials. The scatter of maximum gradient is within $5 \%$ on 5 single cell cavities statistics. The average maximum gradient was $31.4 \pm 1.6 \mathrm{MV} / \mathrm{m}$ with TESLA shape. This performance satisfies enough the EURO-XFEL specification.

\section{Europe}

W.Singer at DESY has developed large grain/single crystal niobium cavity using WC Heraeus material [2]. The cavities are processed with EP and $\mathrm{BCP}$ and they are tested in collaboration with JLAB. A single crystal cavity has been successfully fabricated from a single crystal niobium block, cut out from a very large grain ingot, rolled to a single crystal sheet. The cavity has been tested at JLAB by BCP and reached the critical field $43 \mathrm{MV} / \mathrm{m}$ with a flat $Q_{0}$ vs. $E_{a c c}$ curve. It was demonstrated that BCP can achieve the critical field without any Q-slope on the single crystalline material. DESY has tested large grain cavities processed by EP, and reached the average highest gradient of $34.3 \pm 3.5 \mathrm{MV} / \mathrm{m}$ with statistics of six measurements. This is enough for the EURO-XFEL project. The gradient is close to the $\mathrm{LC}$ specification but the scatter of $10 \%$ is still a problem for the ILC. After this test, they processed the 
cavity with BCP and got a degradation of the high gradient performance, down to 28 $\mathrm{MV} / \mathrm{m}$. More statistics is needed to make conclusion, however, this result suggests that high gradient performance still could be limited by grain boundaries on BCP.

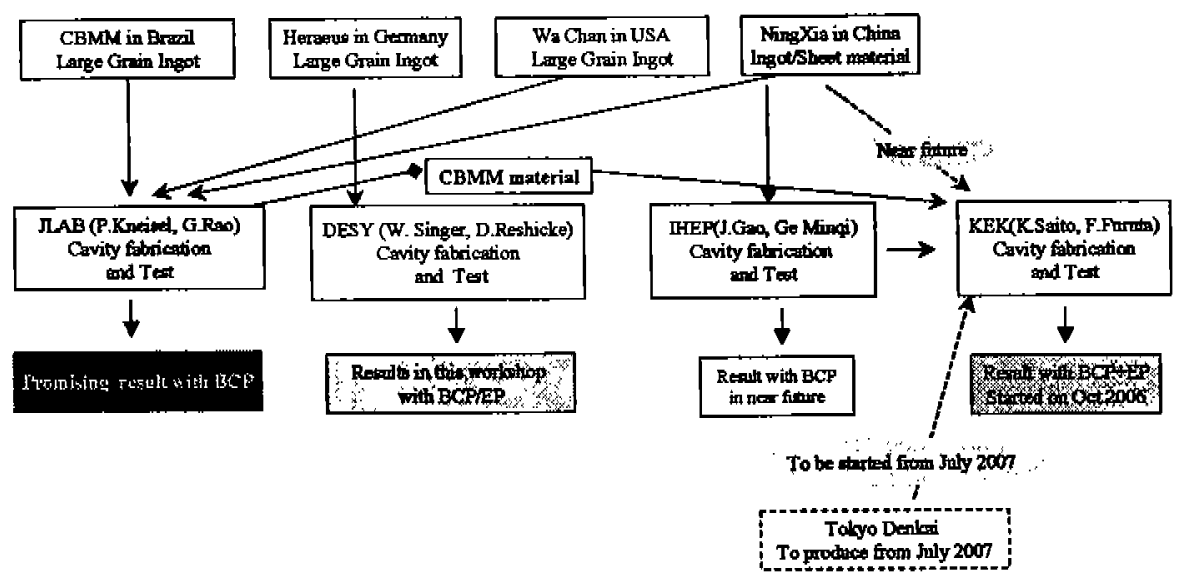

FIGURE 1. Scheme of Large Grain / Single Crystal Niobium Cavity R\&D.

\section{Asia}

Figure 1 shows the current activities in Asia on the large grain niobium cavities. As shown above, NingXia large grain material has been recognized to reach high gradient $\sim 30 \mathrm{MV} / \mathrm{m}$ with BCP. Therefore the China ILC group is eager to push this material as their important contribution to ILC R\&D program. Dr. J.Gao at IHEP has set this issue as their ILC R\&D program. Ge Minqi at IHEP is fabricating 5 single cell cavities using this material. They will test them in IHEP with BCP first, then they will send some of them to KEK, and test using EP.

As will be described later in detail, KEK has fabricated four large grain single cell cavities: one with CBMM material from P. Kneisel and three with NingXia material from J.Gao. We have compared the cavity performance between polycrystalline and these large grain cavities. KEK has a strong mechanical grinding method (Centrifugal Barrel Polishing: CBP) to remove grain boundary steps. The preparation with combined CBP and EP will give unique information about influence of the grain boundary steps on high gradient performance. Tokyo Denkai is also preparing for large grain niobium ingot production. So far they have one suitable EBM furnace for niobium ingot production but the crucible is too small to produce $\sim 300 \mathrm{~mm}$ dia. ingot. They are building two more EBM furnaces for tantalum production by June 2007, then they will use the spare high power EBM furnace for niobium ingot production, which can produce large ingot with $-350 \mathrm{~mm}$ diameter. They will start large grain ingot production in July 2007 [3]. 


\section{KEK ACTIVITY ON LARGE GRAIN NIOBIUM CAVITY}

\section{CBMM Large Grain Cavity}

\section{CBMM Cavity Fabrication}

KEK has received two CBMM large grain niobium sheets (RRR=300) from $Л A B$. Figure 2a shows one of them. These sheets were sliced from CBMM large grain ingot by EDM at JLAB. After cleaning these sheets by light BCP and ultrasonic water rinsing at $\mathrm{KEK}$, we annealed them at $750^{\circ} \mathrm{C}$ for $5 \mathrm{hr}$ in order to release stress, which could be generated during the slicing. We made deep draw them with the same procedure as polycrystalline sheets. The cavity shape is the centre cell shape (LL shape) of Ichiro 9-cell cavity. We did not repress during the deep drawing process, which is usually done at $\mathrm{JLAB}$ to cornect inhomogeneous forming due to grain boundary slipping. As a reference cavity, another single cell cavity (IS\#8) was fabricated in parallel using Tokyo Denkai $(R R R=300)$ polycrystalline material. Figure $2 b$ shows one half-cup from the CBMM large grain sheet after deep drawing. As seen in Fig. 2c, cracks appeared on the inis circle. The half-cups had locally thinner sections on the iris after trimming but could be barely used by a measure in electron beam welding (EBW) as seen later. The equator was wavy due to grain slipping (Fig. 2b). On the other hand, polycrystalline material has very homogeneous forming. After trimming, we measured the shape with a 3D machine. Table 1 shows the results. Large fluctuations from the design value, which is larger by nearly a factor 10 compared to the polycrystalline one, were observed in the large grain half-cups both at the iris and equator. We made EBW of the CBMM large grain cavity in KEK machinery centre. The equator was welded first from the inside with non-full penetration beam, then from the outside. After that a niobium ring $20 \mathrm{~mm}$ long was welded first on the iris by the same EBW method as at the equator (Fig. 2a). This is a measure to avoid EBW failure at the locally thinner locations on the iris (Fig. 2c). Figure 3b shows a picture of the equator EBW seam inside the CBMM cavity. At last, straight beam pipes with titanium flange $60 \mathrm{~mm}$ diameter and $130 \mathrm{~mm}$ long were welded on the cell. Figure $3 \mathrm{c}$ shows the completed CBMM large grain cavity.

TABLE 1. Comparison of circular variation between large grain and polycrystalline.

\begin{tabular}{lcc}
\hline Equator Inside & + Max $(\mathbf{m m})$ & - Max $(\mathbf{m m})$ \\
\hline US No. 1 & 0.4730 & 0.4167 \\
US No. 2 & 0.3866 & 0.2780 \\
Fine 1 & 0.04044 & 0.02941 \\
Fine 2 & 0.0707 & 0.05366 \\
Iris Inside & Max (mm) & $-\mathbf{M a x}(\mathbf{m m})$ \\
\hline US No. 1 & 0.1801 & 0.08088 \\
US No. 2 & 0.1801 & 0.1250 \\
Fine 1 & 0.02805 & 0.02317 \\
Fine 2 & 0.01563 & 0.01443 \\
\hline
\end{tabular}



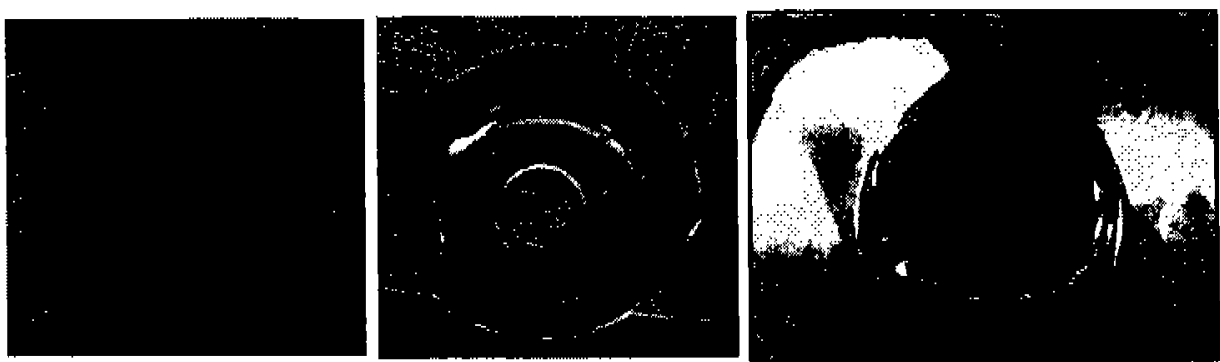

FIGURE 2. CBMM large grain Nb sheet (a) and after deep drawing (b). Cracks were present on the iris section.
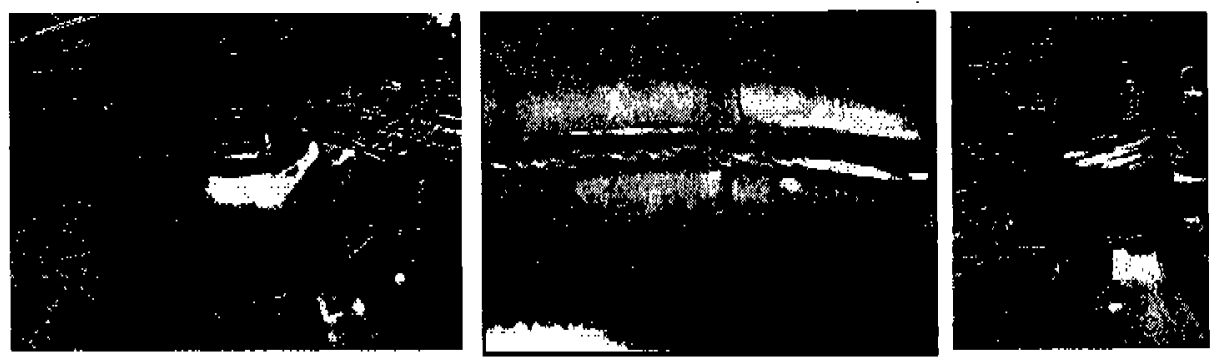

FIGURE 3. Inis EBW (a) inside view of the equatorial EBW seam (b) and completed cavity (c).

\section{CBMM Cavity Preparation}

The cavity was processed with centrifugal barrel polishing (CBP) to remove defects on the cavity inner surface, especially on the equator EBW seam. We have to remove about $200 \mu \mathrm{m}$ by CBP due to the deeper EBW under cut on the equator seam (see Fig. $3 b)$. Figure 4 a shows the equator EBW surface after CBP. Figure $4 \mathrm{~b}$ shows the same surface cleaned by light BCP $(\sim 10 \mu \mathrm{m})$ after CBP. The cavity was annealed at $750^{\circ} \mathrm{C}$ for $3 \mathrm{hr}$ to degas hydrogen picked up during $\mathrm{CBP}$.

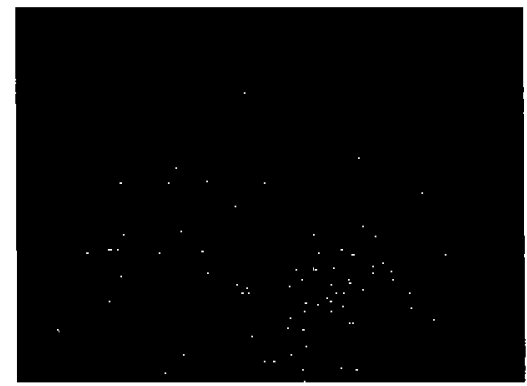

a)

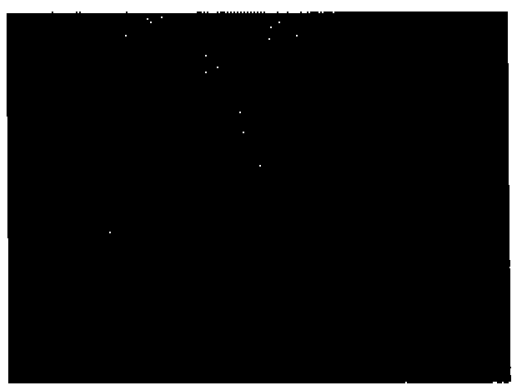

b)

FIGURE 4. Equator EBW seam of CBMM cavity after CBP (a) and after light BCP (b).

After that, it was electropolished for $40 \mu \mathrm{m}$ first, then successively removed $3 \mu \mathrm{m}$ using fresh EP acid closed in the cavity: this procedure was developed recently to have high reliability of high cavity performance. Following this EP process, the cavity was 
rinsed with high-pressure water (HPR) for $1 \mathrm{hr}$ with ultra-pure water $(\mathrm{TOC}=5 \mathrm{ppb}$, Bacteria $=2$ pieces $/ \mathrm{cc}$ ), and dried for one night in the KEK class 10 cleanroom. After assembling, the cavity was vacuum evacuated and baked at $120^{\circ} \mathrm{C}$ for $12 \mathrm{hr}$. The EP material removal and the baking period are different from the KEK standard recipe: EP $(80 \mu \mathrm{m})$, baking $\left(120^{\circ} \mathrm{C}, 48 \mathrm{hr}\right)$. The $12 \mathrm{hr}$ baking period is an input from ЛAB on large grain cavity on BCP large grain cavity. Large grain material might have much less surface defects than polycrystalline cavity. We have tried to reduce EP material removal in this study.

\section{CBMM Cavity RF Test Results}

The cavity RF parameters are summarized in Table 2 . The temperature dependence of surface resistance is presented in Fig. 5 for both cavities. The residual resistance is $1.49 \mathrm{n} \Omega$ on CBMM (USLG) cavity, which is very small as compared to that of the polycrystalline cavity (IS\#8): $7.66 \mathrm{n} \Omega$. As shown in Fig. 6, the gradient of CBMM (USLG) cavity was limited $36.5 \mathrm{MV} / \mathrm{m}$ by quench. On the other hand, it has reached $50 \mathrm{MV} / \mathrm{m}$ with Q-slope on the polycrystalline cavity (IS\#8). We made additional baking on both cavities. The Q-slope in the polycrystalline cavity was eliminated after a total of 48 hr baking, but no effect happened on the CBMM cavity.

TABLE 2. RF Parameters of the CBMM cavity

\begin{tabular}{lc}
\hline RF Parameters & \\
\hline Frequency $(\mathrm{MHz})$ & 1289.519 \\
$\mathrm{R} / \mathrm{Q}(\Omega)$ & 138.6 \\
$\mathrm{E}_{\mathrm{p}} / \mathrm{E}_{\mathrm{Rec}}$ & 2.08 \\
$\mathrm{H}_{\mathrm{p}} / \mathrm{E}_{\mathrm{acc}}[\mathrm{Oe} /(\mathrm{MV} / \mathrm{m})]$ & 35.6 \\
$\Gamma(\Omega)$ & 281.8 \\
$\mathrm{E}_{\mathrm{acd}} /\left(\mathrm{P}_{\mathrm{los}} \mathrm{Q}_{0}\right)^{1 / 2}$ & 100.3 \\
\hline
\end{tabular}

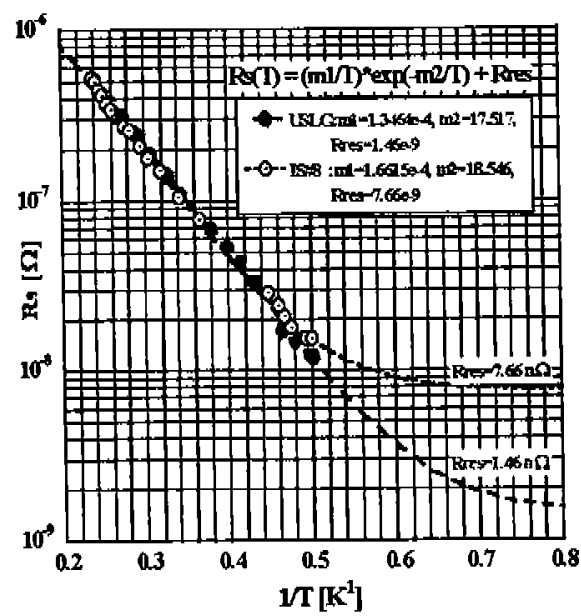

FIGURE 5. T-dependence of surface resistance for the CBMM (USLG) cavity and polycrystalline cavity (IS\#8). 


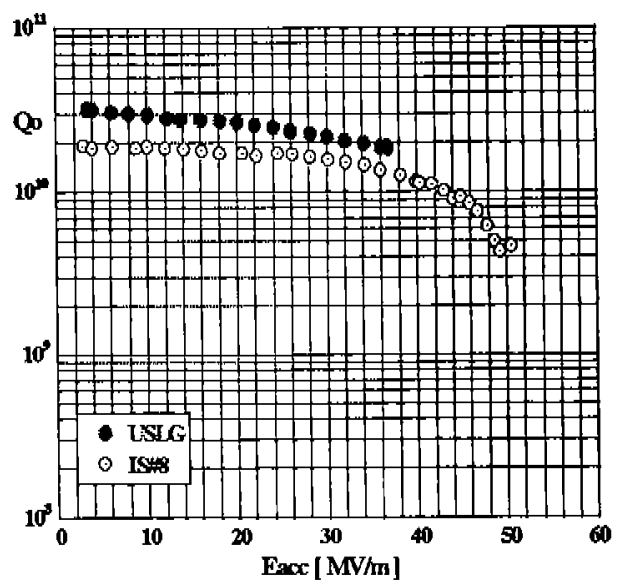

FIGURE 6. RF performance of the CBMM (USLG) cavity and polycrystalline cavity (IS\#8).

\section{Ningxia Large Grain Cavity}

\section{Ningxia Cavity Fabrication}

KEK has received six NingXia large grain niobium sheets with RRR 300 from J. Gao, IHEP in China A representative sheet is shown in Fig.7a. The number of grain is 17 in the NingXia sheet and 10 in the CBMM sheet. The average grain size of the NingXia material $(\sim 6 \mathrm{~cm})$ is $25 \%$ smaller than that of CBMM material $(7.6 \mathrm{~cm})$. We fabricated three Ichiro single cell cavities using these sheets. In the deep drawing process, we met the same cracking problem on the iris circle and wavy shape on the equator section. The fabrication error after the trimming is summarized in Table 3. The fabrication error (maximum fluctuation for inside and outside from design value) of half-cups is similar for both NingXia and CBMM materials. These numbers are quite large compared to the referenced polycrystalline material. Reduction of the big fabrication error would be a future $R \& D$ issue on the large grain cavity. EBW of these cavities is done by the same method as CBMM cavity. A typical EBW seam on equator section is shown in Fig.7b. After completion of the cavities, we removed about $200 \mu \mathrm{m}$ by CBP. Such a heavy material removal is due to the deep EBW under cut on the equator seam or deep "triple tree" grain boundary slipping (see Fig.7b). The CBP final finished surface is shown in Fig.7c. 

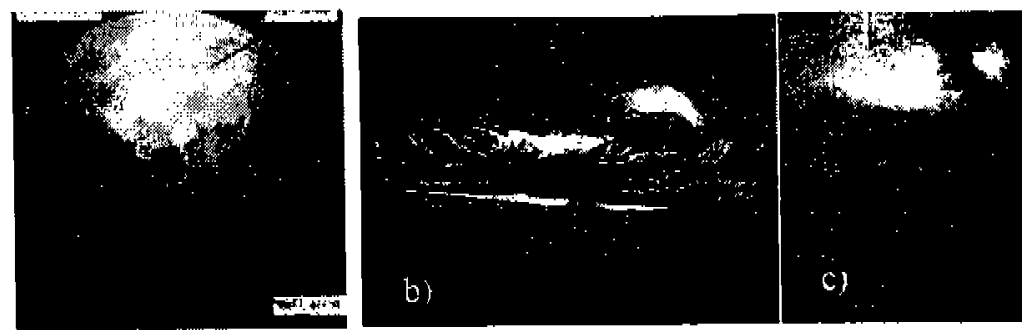

FIGURE 7. NingXia large grain $\mathrm{Nb}$ sheet (a), inside view of the equatorial EBW before (b) and after (c) CBP.

TABLE 3. Fabrication Error in NingXia half-cell cups.

\begin{tabular}{|c|c|c|c|c|c|c|c|c|}
\hline Items: & $\begin{array}{l}\text { Cell } \\
\text { No. }\end{array}$ & $\mathrm{N} \times 2$ & $\mathrm{~N} \times \mathrm{s}$ & NX6 & $\mathrm{N} \times 7$ & NX8 & NX9 & Average \\
\hline \multirow{2}{*}{$\begin{array}{l}\text { 3D are } \\
\text { maide }\end{array}$} & $\max$ & 0 & +0.25 & $: 0.3$ & $+0,3$ & +0.25 & +03 & +0.23 \\
\hline & min & -0.8 & 0.6 & -5.6 & -0.5 & -13 & -0.5 & -0.58 \\
\hline \multirow{2}{*}{$\begin{array}{l}\text { Equlfter } \\
\text { Inside }\end{array}$} & $\max$ & +0.37 & +025 & +0.25 & +0.25 & +0.20 & +0.20 & +025 \\
\hline & $\min$ & -0.25 & -0.18 & -0.13 & -0.13 & -0.18 & -0.20 & -0.18 \\
\hline \multirow{2}{*}{$\begin{array}{l}\text { Equator } \\
\text { Hetght }\end{array}$} & $\max$ & 58.2 & 57.9 & 57.9 & 57.8 & 57.8 & 57.8 & 57.9 \\
\hline & $\min$ & 57.9 & 57.5 & 57.7 & 575 & 57.6 & 57.6 & 57.6 \\
\hline \multirow{2}{*}{$\begin{array}{l}\text { Equator } \\
\text { Surface }\end{array}$} & $\max$ & +0.3 & +0.38 & +0.23 & +0.19 & +0.15 & +0.25 & +025 \\
\hline & $\min$ & 0 & 0 & 0 & 0 & 0 & 0 & 0 \\
\hline \multirow{2}{*}{$\begin{array}{l}\text { Equator } \\
\text { Ontiside }\end{array}$} & $\max$ & +0.25 & +0.55 & +0.38 & +0.15 & $+0,25$ & +0.28 & +0.26 \\
\hline & min & -0.36 & 0.45 & 0.38 & 0.25 & $-0,27$ & -0.38 & -035 \\
\hline \multirow{2}{*}{$\begin{array}{c}\text { Iris } \\
\text { Inside }\end{array}$} & $\operatorname{mex}$ & +0.5 & +0.45 & +0.38 & +443 & +033 & +0.30 & +0.40 \\
\hline & $\min$ & -0.1 & -0.13 & -0.15 & -0.13 & -0.13 & -0.15 & $-\mathbf{0 . 1 3}$ \\
\hline \multirow{2}{*}{$\begin{array}{c}\text { Iris } \\
\text { Surfact }\end{array}$} & $\max$ & +0.05 & +0.06 & +0.05 & +0.07 & +0.06 & +0.06 & $+0,06$ \\
\hline & min & 0 & 0 & 0 & 0 & 0 & 0 & 0 \\
\hline \multirow{2}{*}{ Oritside } & $\max$ & +0.08 & +0.07 & +0.09 & +0.07 & +0.1 & +0.1 & +0.09 \\
\hline & $\min$ & 0,34 & -0.38 & -0.35 & -0.22 & -0.4 & -0.40 & $-0,35$ \\
\hline
\end{tabular}

\section{Ningxia Cavity RF Test Results}

The three NingXia cavities named as CLG\#1, \#2, and \#3 were tested for the following issues: 1) use KEK standard recipe directly and see the high gradient cavity performance, investigate the shorter baking period for example $12 \mathrm{hr}$ at $120^{\circ} \mathrm{C}, 2$ ) investigate material removal effect on cavity performance every $30 \mu \mathrm{m}$ EP after CBP, 3) investigate EP material removal effect on every $30 \mu \mathrm{m}$ without CBP.

The above issue 1) was investigated using CLG\#1 and \#2. These cavities were treated by the KEK current best recipe: CBP, light BCP $(10 \mu \mathrm{m})$, annealing $\left(750^{\circ} \mathrm{C}, 3\right.$ hr), EP $(80 \mu \mathrm{m})+\mathrm{EP}(3 \mu \mathrm{m})$ with fresh acid, HPR $(7 \mathrm{MPa}, 1 \mathrm{hr})$, and bake $\left(120^{\circ} \mathrm{C}\right.$, $48 \mathrm{hr}$ ). In the first preparation for CLG\#2, the $12 \mathrm{hr}$ bake period was tested. The result is shown in Figure 8 . The maximum gradient reached $43.8 \mathrm{MV} / \mathrm{m}$ but $\mathrm{Q}$-slope appeared over than $40 \mathrm{MV} / \mathrm{m}$, so we made an additional $12 \mathrm{hr}$ bake subsequently to 
the test. The maximum gradient did not change by this baking but the Q-slope was rather improved (Fig. 9, triangle sy mbols). We need further study but we judged that the $12 \mathrm{hr}$ bake period is not enough for electropolished large grain cavity. As shown in Fig. 8, we baked directly for $48 \mathrm{hr}$ the cavity CLG\#l and the maximum gradient of $47.9 \mathrm{MV} / \mathrm{m}$ was achieved with rather small Q-degradation, which was due to field emission. Considering those results, we have concluded that the bake period cannot be shortened with electropolished large grain cavity.

The gradients of all NingXia cavities are presented in Fig.8. The highest gradient CLG\#1 has reached is $47.9 \mathrm{MV} / \mathrm{m}$, after a total EP material removal of $99 \mu \mathrm{min}$ three steps. CLG\#2 has reached $43.2 \mathrm{MV} / \mathrm{m}$ with one EP $(80 \mu \mathrm{m}+3 \mu \mathrm{m}$ fresh acid, closed). CLG\#3 has reached $13 \mathrm{MV} / \mathrm{m}$ with the first $33 \mu \mathrm{m} \mathrm{EP}$, without CBP. The residual resistance of the NingXia large grain cavities was between $2.5 \mathrm{n} \Omega$ and $7.5 \mathrm{n} \Omega$. The cavity performance is very similar to the polycrystalline cavities. By recent study, the average gradient of polycrystalline cavities is $46.7 \pm 1.9 \mathrm{MV} / \mathrm{m}$ and residual surface resistance of $\sim 4 \mathrm{n} \Omega$ on the statistics of six cavities [4].

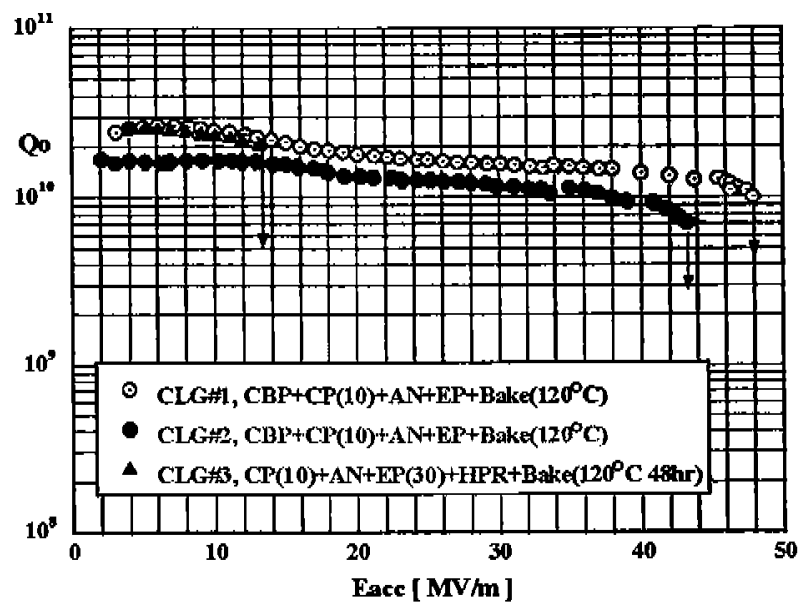

FIGURE 8. High gradient cavity performance using KEK standard recipe. 


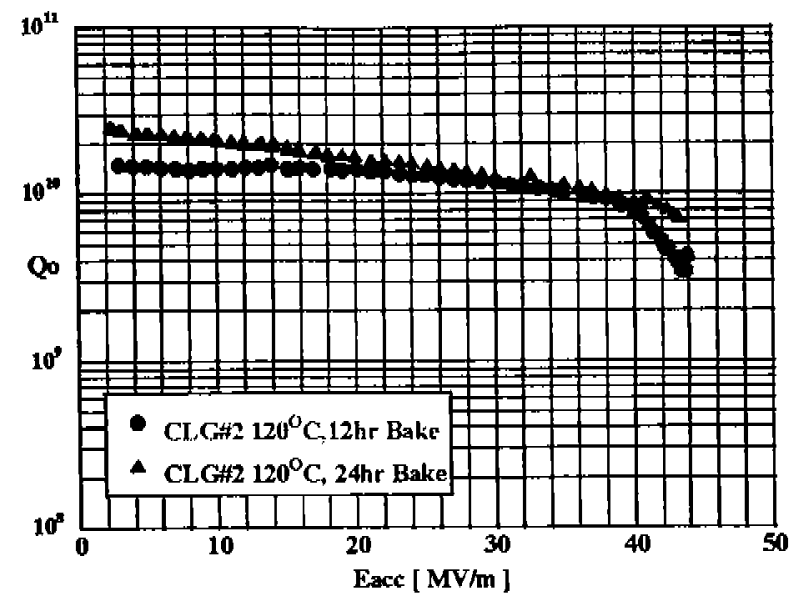

FIGURE 9. RF performance of cavity CLG $\# 2$ after baking for $12 \mathrm{hr}$ and $24 \mathrm{~h}$.

The EP material removal effect after CBP was investigated. CLG\#1 was processed with CBP, which removed the surface about $200 \mu \mathrm{m}$ at the equator region. After that, it was tested after each $33 \mu \mathrm{m}$ EP removal step. In this study, we included the additional $3 \mu \mathrm{m}$ EP using fresh EP acid after normal $30 \mu \mathrm{m}$ EP. This process could avoid the risk of contamination after EP. Figure 10 shows the EP material removal effect on residual surface resistance. The residual surface resistance seems to saturate around $3 \mathrm{n} \Omega$ after $66 \mu \mathrm{m}$ EP. Including other results on NingXia large grain materials, the residual resistance is between $2.5 \mathrm{n} \Omega$ and $7 \mathrm{n} \Omega$. Small residual surface resistance as for the CBMM cavity $(1.46 \mathrm{n} \Omega)$ was not observed for NingXia large grain cavities.

Figure 11 shows the EP material removal effect on the gradient. The gradient has been reached $47.9 \mathrm{MV} / \mathrm{m}$ after $99 \mu \mathrm{m}$ EP. Field emission was the limit in every test. We need further study on this issue, however, a trend is observed that large grain material needs more material removal compared to polycrystalline cavity, if we remind polycrystalline cavity has reached $50 \mathrm{MV} / \mathrm{m}$ after $40 \mu \mathrm{m}$ EP. 


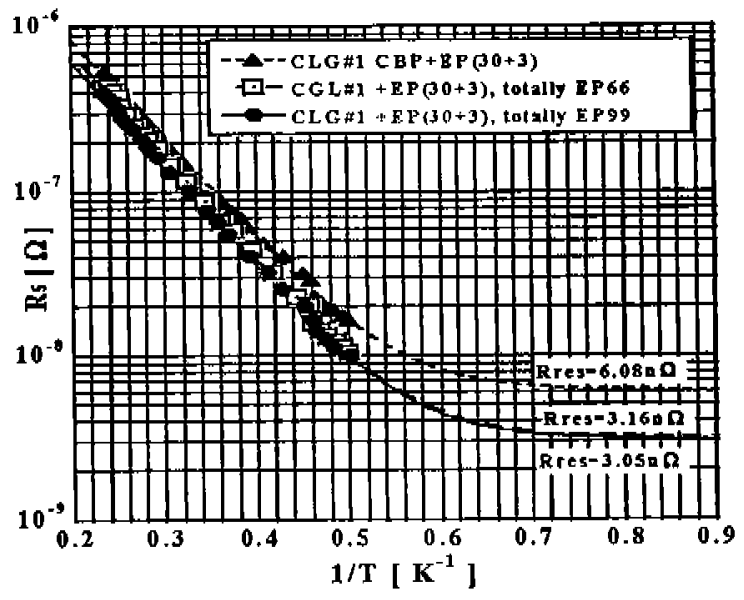

FIGURE 10. Improvement of surface resistance by additional EP.

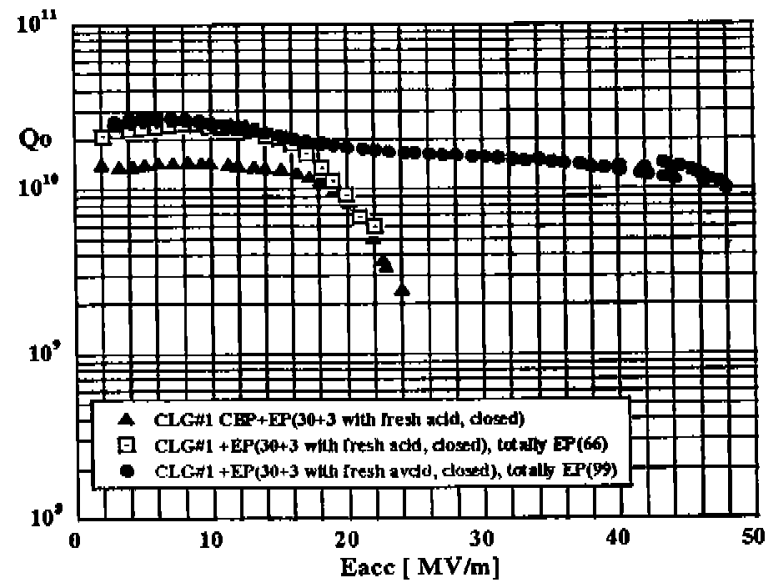

FIGURE 11. Improvement of high gradient performance by additional EP.

\section{FURTHER PROGRAM ON LARGE GRAIN MATERIAL IN JAPAN}

\section{Large Grain Ingot Production in Tokyo Denkai}

As mentioned above, Tokyo Denkai will start large grain niobium ingot production from July 2007 [3]. The crucible of their current electron beam melting furnace for niobium has a $230 \mathrm{~mm}$ diameter and no way to produce $300 \mathrm{~mm}$ diameter ingot. 
Currently they are installing three more electron beam melting furnaces by July 2007 for tantalum production, which is their main business. When they will finish the installation, one furnace with $300 \mathrm{~mm}$ diameter crucible can be used for niobium ingot production.

\section{Development of Wire Sawing Machine}

The large grain niobium material is very promising as seen above. However, the current large grain niobium sheet is not so cheap due to ingot slicing method by EDM (time consuming) or mechanical sawing (lots of waste material). To make realistic the large grain niobium application in cavity production, the development of a high efficiency ingot-slicing method is necessary. In the ILC project, 500 sheets are required every day for three years production. We need a faster ingot slicing macline with less material waste. KEK has a program to develop a niobium ingot-slicing machine by collaboration with a silicon wafer company.

\section{SUMMARY}

KEK was behind from US or Europe on the large grain cavity R\&D and recently started the R\&D by the collaborations with $Л \mathrm{AB}$ and IHEP. We fabricated single cell cavities from CBMM and NingXia large grain niobium sheets and obtained very promising results. On the cavity fabrication, inhomogeneous forming was a problem and has to be solved. Cavity performance of the large grain cavity is similar to the polycrystalline cavity. If material cost is really reduced by this method, it is a good way for mass production such as $\mathrm{ILC}$.

\section{REFERENCES}

1. P.Kneisel et al., this workshop.

2. W. Singer et al., this workshop.

3. K. Takeuchi (private communication).

4. F. Furuta, TTC Meeting at KEK, September 2006. 